\title{
Suppression of a Brownian noise in a hole-type sensor due to induced- charge electro-osmosis
}

Cite as: Phys. Fluids 28, 032003 (2016); https://doi.org/10.1063/1.4943495

Submitted: 05 August 2015 . Accepted: 24 February 2016 . Published Online: 14 March 2016

Hideyuki Sugioka
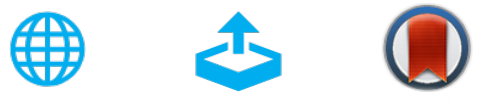

View Online

Export Citation

\section{ARTICLES YOU MAY BE INTERESTED IN}

Rectified motion in an asymmetrically structured channel due to induced-charge electrokinetic and thermo-kinetic phenomena

Physics of Fluids 28, 022004 (2016); https://doi.org/10.1063/1.4941300

Impact of orifice orientation on a finite-span synthetic jet interaction with a crossflow

Physics of Fluids 28, 037106 (2016); https://doi.org/10.1063/1.4943520

Controllable Leidenfrost glider on a shallow water layer

AIP Advances 8, 115209 (2018); https://doi.org/10.1063/1.5051238

\section{Physics of Fluids GALLERY OF COVERS}




\title{
Suppression of a Brownian noise in a hole-type sensor due to induced-charge electro-osmosis
}

\author{
Hideyuki Sugioka ${ }^{a}$ \\ Frontier Research Center, Canon, Inc. 30-2, Shimomaruko 3-chome, Ohta-ku, Tokyo \\ 146-8501, Japan and Department of Mechanical Systems Engineering, Shinshu University \\ 4-17-1 Wakasato, Nagano 380-8553, Japan
}

(Received 5 August 2015; accepted 24 February 2016; published online 14 March 2016)

\begin{abstract}
Noise reduction is essential for a single molecular sensor. Thus, we propose a novel noise reduction mechanism using a hydrodynamic force due to induced-charge electro-osmosis (ICEO) in a hole-type sensor and numerically examine the performance. By the boundary element method that considers both a Brownian motion and an ICEO flow of a polarizable particle, we find that the Brownian noise in a current signal is suppressed significantly in a converging channel because of the ICEO flow around the particle in the presence of an electric field. Further, we propose a simple model that explains a numerically obtained threshold voltage of the suppression of the Brownian noise due to ICEO. We believe that our findings contribute greatly to developments of a single molecular sensor. () 2016 AIP Publishing LLC. [http://dx.doi.org/10.1063/1.4943495]
\end{abstract}

\section{INTRODUCTION}

The hole-type sensor is often introduced as "nanochannels" or "nanopores" and the concept is akin to a Coulter counter that detects the number of blood cells. However, it includes the sensors which hole size is much smaller than that of the Coulter counter and it has a possibility to distinguish the difference of individual molecules. ${ }^{1}$ Namely, as a single molecular sensor, the hole-type sensor has attracted much attention. Obviously, the key of the realization is a noise reduction in a current signal during the passage of a particle through a nano-hole and it is related to the surface phenomena concerning the particle, wall, and electrolyte in confined space. Thus, many researchers propose various models with various methods to obtain the clue. ${ }^{1-5}$ For example, Ghosal ${ }^{2}$ calculated a translocation speed as a function of the pore geometry by using a simple rod model of a charged polymer without considering a Brownian motion and showed the validity by the comparison with the previous experimental data. Jou et al. also calculated a translocation time through a nanopore in a semiconductor membrane by using a chain model (a coarse-grained polyelectrolyte model) of DNA with Brownian dynamics and a Poisson-Nernst-Planck (PNP) model and pointed out that the effect of the surface condition of the nanopore is important. Zwolak and Ventra ${ }^{5}$ also theoretically showed that the four bases included in a DNA molecule provide different electric signals by the application of the transverse electric field to the channel by using a molecular orbital model considering a Green's function method for an electric transportation process across electrodes. However, at least for hole-type sensor, the model that considers a Brownian motion of a particle with a correct surrounding flow considering boundary conditions has not been explored well in spite of their importance, although Brownian dynamics using a chain model $^{3}$ usually consider a viscous drag on a particle for an unbounded problem (i.e., Stokes resistance).

Recently, induced-charge electro-osmosis (ICEO) has been considered to be a key concept for understanding behaviors of polarizable particles in electrolyte. ${ }^{6-9}$ That is, it is recognized that the hydrodynamic interaction due to an ICEO flow around a particle is much more important than the

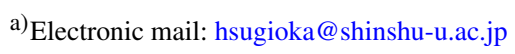


raw electric force such as a dielectrophoretic (DEP) force in an electrolyte. ${ }^{10-12}$ Here, ICEO $^{13,14}$ is caused by the interaction between an electric field $\mathbf{E}$ and ions in an electric double layer formed by the polarizing effect of the electric field. Thus, it generates large flow velocity proportional to $\mathbf{E}^{2}$. Further, in our previous paper ${ }^{15}$ we showed that an attitude and positioning of an elliptical conductive particle is effectively controlled through ICEO effects and thus ICEO plays an important role in a hole-type sensor. However, simulations that consider a Brownian motion with a surrounding ICEO flow have not been explored yet.

Therefore, in this study, we focus on a fundamental hole-type ICEO sensor (of a polarizable particle) that reduces Brownian noise and elucidate its design concept. In particular, by solving the Stokes equation that considers both a random Brownian force and an ICEO effect along with correct boundary condition, we evaluate the detectable current signal during the translocation of a particle through a pore. Further, to understand the noise reduction mechanism well, we will provide a simple model that predicts a threshold voltage of the suppression of the Brownian noise due to ICEO.

This paper is presented in five sections. In Sec. II, we describe methods for a geometry model of a hole-type ICEO sensor, a two-dimensional (2D) flow model, an ICEO slip velocity and DEP force model, a 2D random Brownian force model, a simple model for the suppression of a Brownian noise, and a current signal model. Based on these methods, results of flow fields considering a Brownian noise with an ICEO flow, trajectories and current signals with and without ICEO effects, and other characteristics of the hole-type ICEO sensor are presented in Sec. III. Following a discussion in Sec. IV, our calculations are summarized in Sec. V.

\section{THEORY}

\section{A. Geometry model of a hole-type ICEO sensor}

Figure 1 shows the schematic view of a hole-type sensor that reduces a Brownian noise by using a hydrodynamic force due to ICEO. As shown in Fig. 1, we place a circular conductive particle of radius $c=0.05 w$ in a converging channel of length $L=2.25 w$, inlet width $w=5 \mu \mathrm{m}$, and outlet width $w_{2}=0.4 w$. In this device, by applying an electric voltage $V_{0}$ (e.g., typically $0.4 \mathrm{~V}$ ) between the left- and right-side edges under the existence of the pressure difference $\Delta P\left(\equiv P_{1}-P_{2}\right)$ (e.g., $\left.2 \mathrm{~Pa}\right)$ in water, we can detect a signal of an ion current $I_{0}$ during the translocation of the particle through the converging channel. Because of the hydrodynamic repulsion force from wall due to ICEO, the particle moves to the center of the channel ${ }^{15}$ and thus the noise due to a random walk of the Brownian particle is also reduced.

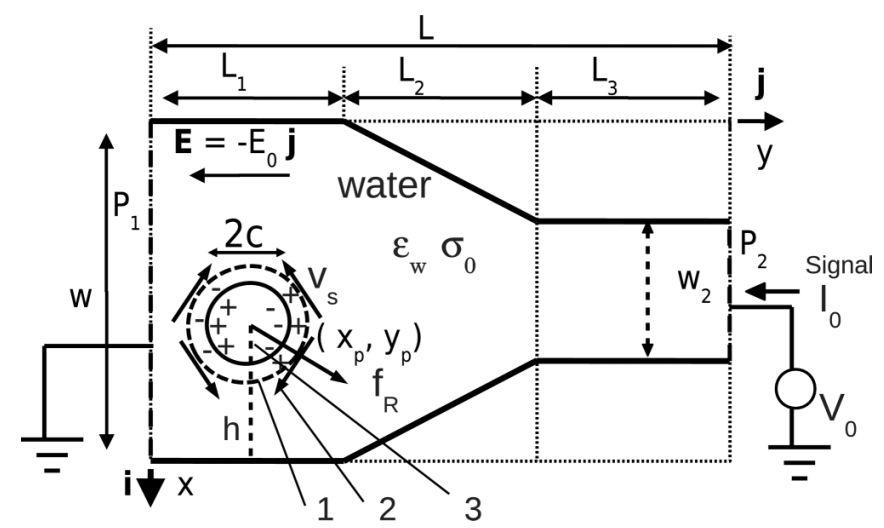

FIG. 1. Schematic view of a hole-type sensor that reduces a Brownian noise by using a hydrodynamic force due to ICEO. 1 -electro double layer. 2-ICEO slip velocity. 3-conductive particle. We place a conductive particle of radius $c=0.05 w$ in a converging channel of length $L=2.25 w$, inlet width $w=5 \mu \mathrm{m}$, and outlet width $w_{2}=0.4 w$. Here, the applied voltage $V_{0}$ is $0.4 \mathrm{~V}$, the pressure difference $\Delta P\left(\equiv P_{1}-P_{2}\right)$ is $2 \mathrm{~Pa}$, the step time period $\Delta t$ is $0.05 \mathrm{~ms}$, and the viscosity $\mu$ is $1 \mathrm{mPa}$. 


\section{B. 2D flow model}

In the microfluidic channel, the Reynolds number $R e$ is usually very small and thus we consider a 2D quasi-static Stokes flow with a Brownian motion and a surrounding ICEO flow of a conductive particle. That is, based on the boundary element method (BEM) along with a thin-double layer approximation, ${ }^{12,15,16}$ we numerically solve the Stokes equation with correct boundary conditions as follows:

$$
\begin{gathered}
\mu \nabla^{2} \boldsymbol{v}-\nabla p=0, \quad \nabla \cdot \boldsymbol{v}=0, \\
\text { On } S_{p}^{+}: \quad \boldsymbol{v}=\boldsymbol{U}+\boldsymbol{V}_{\mathrm{s}}, \\
\int_{S_{p}^{+}} \boldsymbol{f} d l+\boldsymbol{F}^{e x t}=0 \text { (on the particle), } \boldsymbol{v}=0 \text { (on the wall), }
\end{gathered}
$$

where $p$ is pressure, $\mu(\sim 1 \mathrm{mPa})$ is the viscosity, $S_{p}^{+}$denotes the surface defined as the outer edge of the double layer, $\boldsymbol{v}$ is the velocity of the fluid, $\boldsymbol{U}$ is the velocity of the particle, and $\boldsymbol{f}$ is the surface traction that is the force per area exerted on the surface. Further, $\boldsymbol{V}_{s}$ is a slip velocity on $S_{p}^{+}$due to ICEO, $\boldsymbol{F}^{\text {ext }}\left(=\boldsymbol{F}^{R}+\boldsymbol{F}^{D E P}\right)$ is the total external force on the particle, $\boldsymbol{F}^{R}$ is a random Brownian force, and $\boldsymbol{F}^{D E P}$ is a total DEP force. Furthermore, the height of the circular cylinder is $d$ and we set $d=1$ unit length $(1 \mathrm{~m})$ in our 2D problems. Please note that in the BEM, the Stokes equation of Eq. (1) is transformed into the matrix equation ${ }^{16}$ that $[T]\{v\}+[G]\{f\}=0$, where $\{v\}$ and $\{f\}$ are the node vectors of the velocity and surface traction, respectively, and the boundary conditions of Eqs. (2) and (3) provide the limitation for $\{v\}$ and $\{f\}$; thus, we can calculate all the values of $\{v\}$ and $\{f\}$. In particular, $\int_{S_{p}^{+}} f d l+\boldsymbol{F}^{e x t}=0$ in Eq. (3) is a well-known force balance condition for the particle. Although it does not include the velocity, it represents the balance between the viscous force concerning the particle velocity $\boldsymbol{U}$ and the external force $\boldsymbol{F}^{\text {ext }}$ through the Stokes equation of Eq. (1) with the boundary condition of Eq. (2), in the case of $\boldsymbol{V}_{s}=0$. Of course, in the case of $\boldsymbol{V}_{s} \neq 0$, Eqs. (1)-(3) provide a complete formulation for the boundary value problem considering ICEO phenomena.

\section{ICEO slip velocity and DEP force model}

On the basis of the Helmholtz-Smoluchowski formula, the ICEO slip velocity $\boldsymbol{V}_{s}$ for a bounded problem $^{12}$ at each time step is provided as

$$
\boldsymbol{V}_{s}=-\frac{\epsilon_{w} \zeta}{\mu} \boldsymbol{E}_{\mathrm{s}}
$$

where $\boldsymbol{E}_{s}\left(=-\nabla \phi_{0}\right)$ is the tangential electric field on $S_{p}^{+}, \epsilon_{w}\left(\sim 80 \epsilon_{0}\right)$ is the dielectric permittivity of the solvent (typically water), $\epsilon_{0}$ is the vacuum permittivity, $\zeta\left(\equiv \phi_{i}-\phi_{o}\right)$ is the zeta-potential, and $\boldsymbol{n}$ is the surface normal unit vector. Further, $\phi_{o}$ and $\phi_{i}$ are the potentials at the outside- and inside-edges of the double layer, respectively, and $\phi_{o}$ is calculated by solving the Laplace equation

$$
\nabla^{2} \phi_{0}=0
$$

under the condition that $\boldsymbol{n} \cdot \nabla \phi_{0}=0$ on $S_{p}^{+}$, while $\phi_{i}$ is calculated by solving the Laplace equation under the iso-potential condition on the surface of the particle with no charge condition. Please note that as explained the slip velocity for an unbounded problem is provided as ${ }^{7} \boldsymbol{V}_{s}=2 U_{0} \sin 2\left(\frac{\pi}{2}-\theta\right) \hat{\boldsymbol{\theta}}$, where $U_{0}=\epsilon E_{0}^{2} c / \mu, \theta$ is the angle between the $x$ direction and the radial direction, and $\hat{\boldsymbol{\theta}}$ is an unit vector in the angular direction. The expression is approximately correct even for the ICEO problem in a channel if the distance between the wall and the particle is enough large. However, it is not suitable when we consider the precise interaction between the wall and the particle. ${ }^{12}$ Thus, we need to return to the Helmholtz-Smoluchowski formula of Eq. (4) to calculate the slip velocity that considers the boundary effect precisely. Further, we obtain the DEP traction due to the Maxwell stress as

$$
\boldsymbol{F}^{D E P}=\int_{S_{p}^{+}}\left(-\frac{1}{2} \epsilon \boldsymbol{E}_{s}^{2}\right) \boldsymbol{n} d l .
$$

Please note that in our calculations we consider both the ICEO and DEP effects, since generally they are considered to be important in electrokinetic systems. ${ }^{10}$ 


\section{2D random Brownian force}

The variance of the random walk of the Brownian particle during a time period $\Delta t$ (typically, $0.05 \mathrm{~ms})$ is described as ${ }^{17}$

$$
\sigma^{2}(\Delta t)=\left\langle x^{2}(\Delta t)\right\rangle-\langle x(\Delta t)\rangle^{2}=2 D \Delta t,
$$

where $D$ is the diffusivity of the particle, $k$ is the Boltzmann constant, $T$ is the absolute temperature, and $x(\Delta t)$ represents one dimensional motion, and thus the root mean squares (RMS) of the position, velocity, and force of the Brownian motion are described as $\bar{x}(\Delta t)=\sqrt{2 D \Delta t}, \bar{v}_{x}(\Delta t)=\sqrt{2 D \Delta t} / \Delta t$, and $\bar{F}_{x}(\Delta t)=\xi \sqrt{2 D \Delta t} / \Delta t$. Here, $\xi$ is the friction coefficient. Thus, the RMS of the 3D Brownian force is described as $\bar{F}_{x}{ }^{R, 3 D}(\Delta t)=\xi \sqrt{\frac{2 D}{\Delta t}}$. Here, exactly speaking, as pointed out by Schumacher and van de $\mathrm{Ven}^{18}$ in the context of fixed charge particles, the Stokes-Einstein expression for the diffusivity $(D=k T / 6 \pi \mu c)$ might not be valid in an electrolyte solution, due to deformation of the induced Debye cloud, and thus, Stokes's law of resistance $(\xi=6 \pi \mu c)$ also might not be reliable for the particle that has an electric double layer. Nevertheless, at the best of my knowledge, the researchers ${ }^{1-3,5}$ usually neglect the above effect and use the well-known relations $(D=k T / 6 \pi \mu c$ and $\xi=6 \pi \mu c)$ for a 3D particle as a rational approximation. Thus, as a first step, we also neglect the above effect and consider that

$$
\bar{F}_{x}^{R, 3 D}(\Delta t)=6 \pi \mu c \sqrt{\frac{2 D}{\Delta t}} .
$$

However, since there is no relation corresponding to Stokes's law of resistance for a $2 \mathrm{D}$ problem, $\xi$ cannot be determined theoretically for a 2D particle in spite of its practical importance. Please note that this is the problem related to the well-known 2D Stokes's paradox at the far distance flow field. Thus, as the first approach, we assume that the 2D circular cylinder and 3D sphere of the density $\rho$ and velocity $V$ have the same drag coefficient $c_{D}$. Namely, under this assumption, we obtain the following relation:

$$
\frac{d \bar{F}_{x}^{R, 2 D}}{\bar{F}_{x}^{R, 3 D}}=\frac{\frac{1}{2} \rho V^{2} S^{2 D} c_{D}}{\frac{1}{2} \rho V^{2} S^{3 D} c_{D}}=\frac{2 d}{\pi c},
$$

where $S^{2 D}=2 d c$ and $S^{3 D}=\pi c^{2}$ are the cross-sectional areas of 2D and 3D particles, respectively. Please note that the above assumption is justified and useful in our bounded system if we can observe a similar Brownian motion which variance is approximately $2 D \Delta t$, as checked in Fig. 4(a) later. From Eqs. (8) and (9),

$$
\bar{F}_{x}^{R, 2 D}=12 \mu \sqrt{\frac{2 D}{\Delta t}}
$$

and thus we obtain the $2 \mathrm{D}$ random Brownian force at each time step as

$$
F_{i}^{R, 2 D}=12 \mu \sqrt{\frac{24 D}{\Delta t}} N_{i},(i=x, y)
$$

where $N_{i}$ is a random number that is uniformly distributed on the interval $[-0.5,0.5]$ and have a variance $\left\langle N_{i}^{2}\right\rangle=\frac{1}{12}$.

\section{E. Simple model for the suppression of a Brownian noise}

The 2D hydrodynamic repulsion force acting on the circular cylinder from the wall at the distance $h$ (in Fig. 1) is described as ${ }^{19}$

$$
F_{h}=4 \pi B \epsilon_{w} c E_{0}^{2} \simeq 40 \pi \epsilon_{w} c E_{0}^{2}(\text { at } h / a=2),
$$

where $B$ is generally a decreasing function of $h / a$, and $B \simeq 10$ at $h / a=2$. Thus, if we can neglect the effect of another wall, we can predict the threshold voltage $V_{0}^{t h}$ by considering the relation that $\bar{F}_{x}^{R, 2 D}=F_{h}$. That is, to suppress the Brownian force under the condition that $h / a \leq 2$, we need to apply a voltage more than the threshold voltage that 


$$
V_{0}^{t h} \simeq L \sqrt{\frac{12 \mu}{40 \pi \epsilon_{w} c} \sqrt{\frac{2 D}{\Delta t}}} .
$$

However, at the center of the channel, we cannot neglect the repulsion force due to another wall. Thus, the repulsion force becomes zero at the center, while it increases rapidly near the wall.

\section{F. Current signal model}

Here, we explain a calculation method to obtain a current signal from the numerical results using the BEM. That is, under the assumption of the thin double layer approximation, we can assume that there is no space charge in the bulk region. Thus, we can neglect a current due to the flow of the space charge, although it becomes important when $\lambda_{D}$ is comparable to $w$, i.e., for the calculation of the current signal, we can use the ordinary bulk electrical conductivity $\sigma_{0}$ due to ion electro-migration. ${ }^{20}$ Thus, we can simply calculate a current signal $I_{0}$ of the hole type sensor just by integrating the current density $\boldsymbol{i}=\sigma_{0} \boldsymbol{E}$ over the $x$ and $z$ directions at the plane of the inlet (at $y=0$ ), i.e., by using the numerical results of $(\nabla \phi)^{y=0}$, we obtain the current signal as

$$
I_{0}=d \sigma_{0} \int_{x=0}^{x=w}(\nabla \phi)^{y=0} d x
$$

where $\sigma_{0}=2(z e)^{2} C_{0} D_{0} / k T=\epsilon_{w} D_{0} / \lambda_{D}^{2},{ }^{20} z e$ is an ion charge, $C_{0}$ is a bulk concentration of ions, $D_{0}$ is the diffusibility of ions, $k$ is the Boltzmann constant, $T$ is the absolute temperature, and $\lambda_{D}=$ $\sqrt{\epsilon_{w} k T / 2(z e)^{2} C_{0}}$ is the Debye screening length.

\section{RESULTS}

\section{A. Flow fields considering a Brownian noise with an ICEO flow}

Figure 2 shows flow fields considering a Brownian motion with an ICEO flow [(a) to (c)] and without the ICEO flow (d). On the one hand, as shown in Figs. 2(a) and 2(b), the Brownian motion induces macroscopic strong random flow fields because of the viscous flow in the narrow channel even if we consider an ICEO flow at $V_{0}=0.4 \mathrm{~V}$; however, as shown in Fig. 2(c), the position of the Brownian motion is restricted in the narrow region near the center of the pore because of the ICEO hydrodynamic repulsion force from the upper and lower walls. On the other hand, in the case of

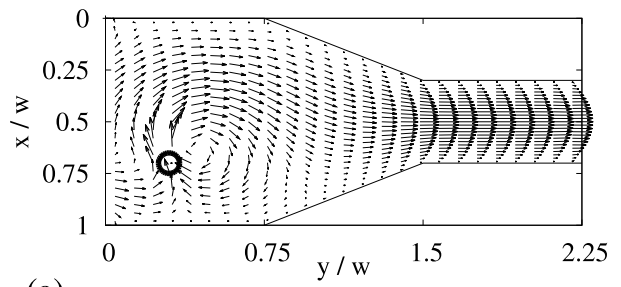

(a)

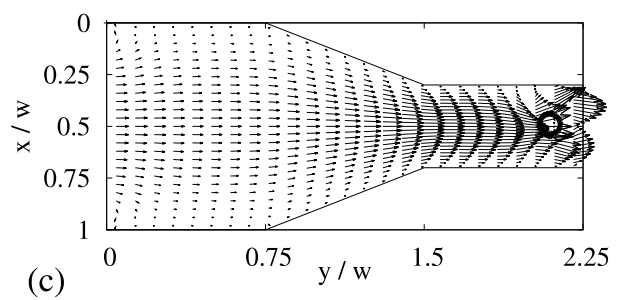

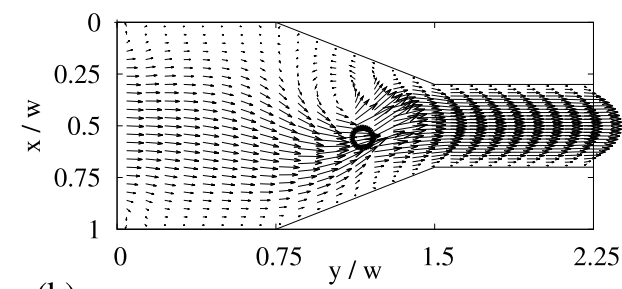

(b)

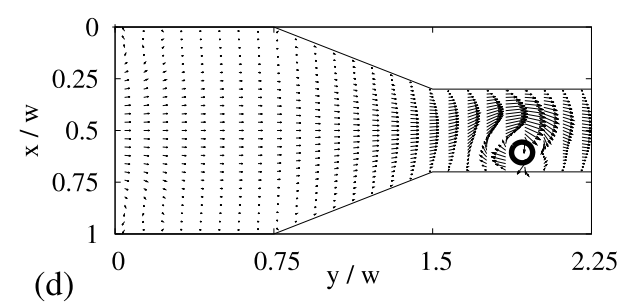

FIG. 2. Flow fields considering a Brownian noise with an ICEO flow [(a) to (c)] and without the ICEO flow (d). Here, $w=5 \mu \mathrm{m}, c / w=0.05, L / w=2.25, w_{2} / w=0.4, \mu=1 \mathrm{mPa} \mathrm{s}, \Delta t=0.05 \mathrm{~ms}, \Delta P=2 \mathrm{~Pa}$, and $x_{p}^{\text {initial }} / w=0.7 ; V_{0}=0.4 \mathrm{~V}$ in (a) to (c), while $V_{0}=0 \mathrm{~V}$ in (d); $t=0,50,90$, and 110 in (a), (b), (c), and (d), respectively. (a) Flow field at $t=0 \mathrm{~ms}\left(V_{0}=0.4 \mathrm{~V}\right)$. (b) Flow field at $t=50 \mathrm{~ms}\left(V_{0}=0.4 \mathrm{~V}\right)$. (c) Flow field at $t=90 \mathrm{~ms}\left(V_{0}=0.4 \mathrm{~V}\right)$. (d) Flow field at $t=110 \mathrm{~ms}\left(V_{0}=0 \mathrm{~V}\right)$. 

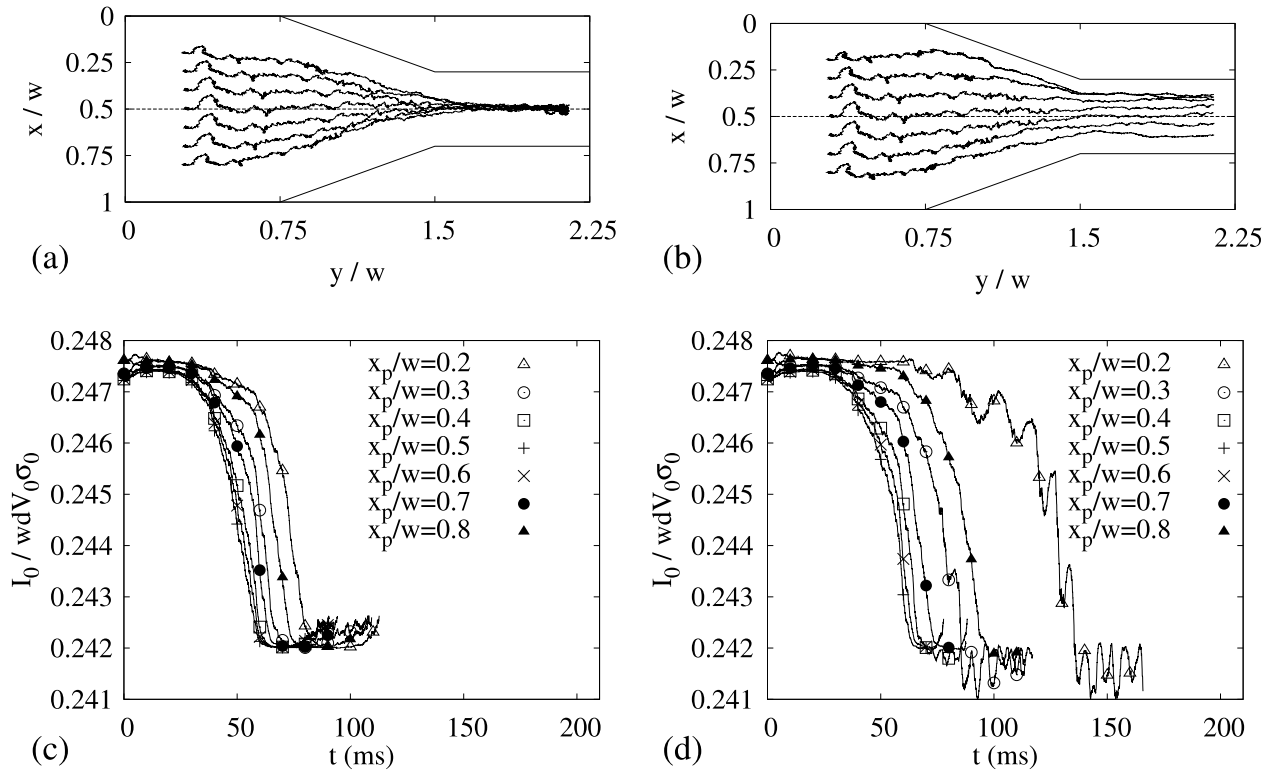

FIG. 3. Trajectories and current signals with and without ICEO effects. Here, $w=5 \mu \mathrm{m}, c / w=0.05, L / w=2.25$, $w_{2} / w=0.4, x_{p}^{\text {initial }} / w=0.7, \mu=1 \mathrm{mPa} \mathrm{s}, \Delta t=0.05 \mathrm{~ms}$, and $\Delta P=2 \mathrm{~Pa}$; in (a) and (c) $V_{0}=0.4 \mathrm{~V}$, while $V_{0}=0 \mathrm{~V}$ in (b) and (d). (a) Trajectory with ICEO $\left(V_{0}=0.4 \mathrm{~V}\right)$. (b) Trajectory without ICEO $\left(V_{0}=0 \mathrm{~V}\right)$. (c) Current signal with ICEO $\left(V_{0}=0.4 \mathrm{~V}\right)$. (d) Current signal without ICEO $\left(V_{0}=0 \mathrm{~V}\right)$.

no ICEO effect at $V_{0}=0 \mathrm{~V}$, the position of the particle is not controlled in the pore because of the Brownian random walk, as shown in Fig. 2(d).

\section{B. Trajectories and current signals with and without ICEO effects}

Figures 3(a) and 3(b) show the trajectories of the particle with different initial positions with and without ICEO effects, respectively, while Figs. 3(c) and 3(d) show the corresponding signals of the measurable current predicted by Eq. (14) with and without ICEO effects, respectively. As shown in Fig. 3(a), the trajectories using ICEO $\left(V_{0}=0.4 \mathrm{~V}\right)$ are limited in the very narrow region near the center due to ICEO effects in spite of their different initial positions, and thus the curves of the corresponding signals become similar, as shown in Fig. 3(c), although the approaching time of the particle to the pore is different each other. Further, as shown in Fig. 3(b), the trajectories not using ICEO $\left(V_{0}=0 \mathrm{~V}\right)$ spread in the channel because of the fluctuation of the Brownian motion, and the magnitude of the corresponding Brownian noise due to the particle passing near the wall becomes much larger than that using ICEO and it increases a total noise level; however, the magnitude of the Brownian noise due to the particle passing near the center is similar to that using ICEO, as shown in Fig. 3(d). It should be noted that we use the same series of random numbers for their comparison.

\section{Other characteristics of the hole-type ICEO sensor}

Figure 4 shows other characteristics of the hole-type ICEO sensor. In Fig. 4(a), we show that the fluctuation of the random walk of radius $c / w=0.05$ at $V_{0}=0 \mathrm{~V}$ under the condition that $w=5$ to $50 \mu \mathrm{m}$. As shown in Fig. 4(a), the fluctuation of the Brownian walk decreases as the value of $w$ increases and it almost vanishes at $w=50 \mu \mathrm{m}$, i.e., at $2 c=5 \mu \mathrm{m}$. These results are very reasonable and thus we believe that our 2D Brownian model is useful as the first attempt. Further, Fig. 4(b) shows an average flow velocity at the inlet. As shown in Fig. 4(b), the average flow velocity fluctuates largely due to the macroscopic random flow fields induced by the Brownian motion [in Figs. 2(a) and 2(b)]. Generally, the flow fluctuation is important when we consider the Brownian motion in the viscous 

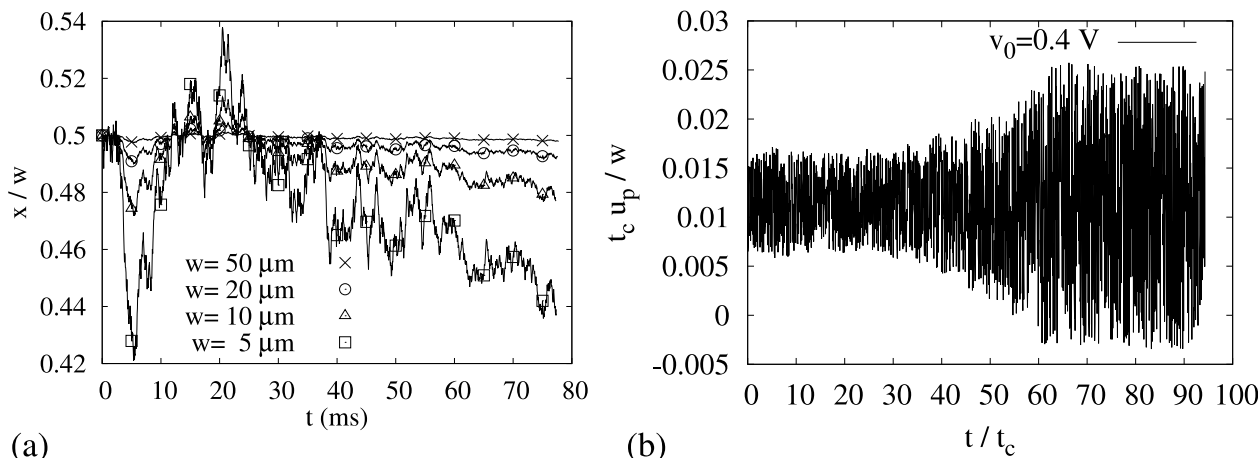

(a)

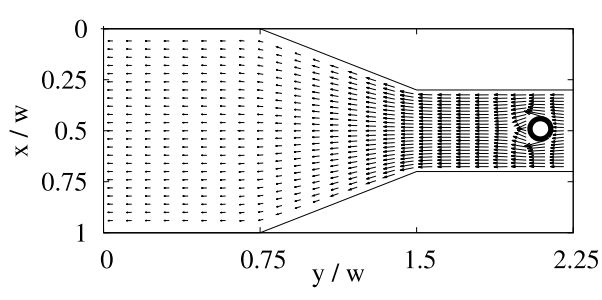

(c)

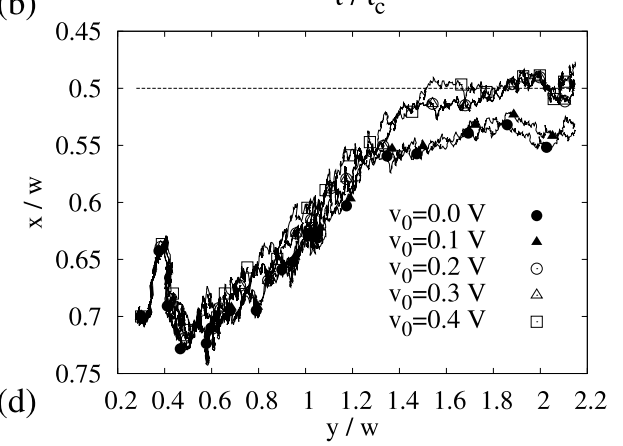

FIG. 4. Other characteristics of the hole-type ICEO sensor. Here, $w=5 \mu \mathrm{m}, c / w=0.05, L / w=2.25, w_{2} / w=0.4$, $x_{p}^{\text {initial }} / w=0.7, \mu=1 \mathrm{mPa} \mathrm{s}, \Delta t=0.05 \mathrm{~ms}, t_{c}=1 \mathrm{~ms}$, and $\Delta P=2 \mathrm{~Pa}$. (a) Fluctuation of the Brownian walk at $w=5-50 \mu \mathrm{m}$ $\left(v_{0}=0.0 \mathrm{~V}\right)$. (b) Fluctuation of the average flow velocity at the inlet $\left(v_{0}=0.4 \mathrm{~V}\right)$. (c) Electric field at $t=90 \mathrm{~ms}\left(v_{0}=0.4 \mathrm{~V}\right)$. (d) Trajectories of the particle at $V_{0}=0-0.4 \mathrm{~V}$.

fluid, although it does not affect on the current noise in the framework of thin double layer approximation as far as $w$ is not so small (as mentioned in Sec. II D). Besides, please note that the fluctuation becomes large when the particle moves in the pore approximately at $t>30 \mathrm{~ms}$. The increasing of the flow fluctuation means the increasing of the drag force of the particle due to the viscosity in the pore, and it suggests that the Brownian motion is also suppressed by the viscosity to some extent within the small bounded space. In addition, Fig. 4(c) shows the electric field corresponding to the flow field at $t=90 \mathrm{~ms}$ in Fig. 2(c). From Fig. 4(c), we understand that the fluctuation of the particle near the center of the pore does not affect largely on the electric field at $y=0$ since the electric resistance does not so change by the fluctuation, and thus the Brownian noise in the current signal is suppressed as mentioned before. Furthermore, Fig. 4(d) shows the trajectories of the particle at different voltages. As shown in Fig. 4(d), the trajectories of the particle at $V_{0}=0.2-0.4 \mathrm{~V}$ are restricted near the center region due to ICEO effect at $y / w>1.5$, while the trajectories at $V_{0}=0$ and $0.1 \mathrm{~V}$ are not restricted near the center region; thus, we can find that the threshold voltage is $0.1 \mathrm{~V}$. Please note that from our simple model in Sec. II E, we can calculate that $D=0.879 \times 10^{-12} \mathrm{~m}^{2} / \mathrm{s}, \bar{F}_{x}{ }^{R, 2 D}=2.25 \mu \mathrm{N} / \mathrm{m}$, and $V_{0}^{t h}=0.11 \mathrm{~V}$ under the condition that $w=5 \mu \mathrm{m}, c / w=0.05, \mu=1 \mathrm{mPa}$, and $\Delta t=0.05 \mathrm{~ms}$; thus, our calculation is reasonable.

\section{DISCUSSION}

\section{A. Meaning of the hole-type ICEO sensor}

By solving the Stokes equation that consider a 2D Brownian force of a polarizable particle with a surrounding ICEO flow in the bounded converging channel, we find that the Brownian motion of the particle is restricted in the center region due to the ICEO flow and thus the corresponding Brownian noise in the detectable current is also reduced. Here, since the suppression of the Brownian noise results from the hydrodynamic repulsion force due to the ICEO flow between the wall and particle, the ICEO flow reduces an effective hole radius corresponding to the magnitude of the flow. Thus, 
the magnitude of the ICEO flow changes the magnitude of the variance of the Brownian noise of the current during the translocation process of a particle in a nanopore channel. In other words, we find that the variance due to the Brownian noise also includes information on the particle through the magnitude of the ICEO flow velocity. Please note that although we showed that attitude and positioning of elliptical conductive particles are controlled by the ICEO effects in our previous paper, ${ }^{15}$ the current signal and the Brownian noise are not considered as a hole-type sensor in the previous paper. Thus, in this paper, we have first clarified the noise reduction mechanism using ICEO in a hole-type sensor.

\section{B. About a 2D Brownian system}

Further, from the viewpoint of numerical calculation technique, we first describe the new implicit method to solve the 2D Brownian system that consider the ICEO flow with proper boundary conditions for the hole-type sensor. Please note that usually in the chain model ${ }^{2}$ viscosity of the fluid is just introduced through the friction coefficient $(\xi)$, while the Brownian motion is neglected in the simple rod model, ${ }^{3}$ as mentioned before. In addition, our simple analytical model provides a useful threshold voltage that suppresses a Brownian noise by the help of the analytical formulation of the ICEO repulsion force provided by Zhao and Bau. ${ }^{19}$ Thus, we believe that our 2D Brownian models clarify the design concept of the hole-type ICEO sensor better than the 3D model as the first approach, since in the 3D model we probably need to care the precision of the numerical results more.

\section{About the role of rotational Brownian motion}

In general, rotational Brownian motion plays an important role to understand the characteristics of anisotropic particles,${ }^{21}$ e.g., dielectric and Kerr-effect relaxation of polar fluids, dielectric relaxation of nematic liquid crystals, and magnetization relaxation of ferrofluids. Even for the hole-type sensor, the knowledge of the rotational Brownian motion of an isotropic particle (e.g., an elliptical conductive particle in 2D problems) is useful for improving the signal-to-noise ratio since the rotational Brownian motion may produce a significant current noise, and thus the suppression of the current noise due to the rotational Brownian noise also becomes important. Fortunately, since the attitude of the elliptical particle is also controlled in the flow direction by the torque due to the ICEO flows, as shown in our previous paper, ${ }^{15}$ we can expect the suppression of the noise due to the rotational Brownian motion through ICEO. Thus, one important approach in the future might be to simulate the rotational Brownian motion for the rigid elliptical conductive particle and to examine the suppression effect due to ICEO.

\section{Hydrodynamic pseudo-potentials due to Brownian motion}

The motivation for our work is a noise reduction in a hole-type sensor. However, we can view this manuscript as more fundamentally considering nanopore motion of polarizable (especially metal) nanoparticles, and even in this general context, our model is attractive. In particular, it seems as though the description of Brownian motion as simply a random force is missing the effect of viscous flows associated with such motion under strong confinement. This can lead to "hydrodynamic pseudopotentials" as described by Squires. ${ }^{22}$ That is, although the hydrodynamic interaction term between beads is usually not considered in a chain model using the so-called Brownian dynamics, ${ }^{3,23}$ our results suggest that the hydrodynamic interaction term due to Brownian motion between beads is important in the chain model, and it should be considered as a hydrodynamic pseudo-potential under strong confinement in the future if we do not calculate hydrodynamic interactions directly.

\section{CONCLUSION}

In conclusion, we have proposed a new mechanism to reduce a Brownian noise in a hole-type sensor owing to the hydrodynamic force due to induced charge electro-osmosis and numerically 
examined the performance. By the multi-physics simulation technique that considers both an ICEO flow and a Brownian motion, we find that (1) the Brownian motion is restricted in the narrow region owing to a hydrodynamic repulsion force from the wall in the confined channel and thus the Brownian noise in the current is suppressed. (2) The surrounding ICEO flow is much more important than the size itself, i.e., the surrounding ICEO flow changes the translocation time, magnitude, and shape of the current; thus, we have a possibility to differentiate individual particles from the translocation current, through the knowledge concerning ICEO phenomena. (3) It also changes the effective radius of the particle in the pore and affects directly on the variance of the noise; thus, the variance of the signal also includes information that differentiates particles. We believe that our findings contribute greatly to developments in studies on a single molecular sensor.

${ }^{1}$ M. Zwolak and M. D. Ventra, "Coloquium: Physical approaches to DNA sequencing and detection,” Rev. Mod. Phys. 80, 141 (2008).

${ }^{2}$ S. Ghosal, "Electrophoresis of a polyelectrolyte through a nanopore," Phys. Rev. E 74, 041901 (2006).

${ }^{3}$ I. A. Jou, D. V. Melnikov, C. R. Mckinney, and M. E. Gracheva, "DNA translocation through a nanopore in a single-layered doped semiconductor membrane," Phys. Rev. E 86, 061906 (2012).

${ }^{4}$ H. W. de Haan and G. W. Slater, "Translocation of a polymer through a nanopore across a viscosity gradient," Phys. Rev. E 87, 042604 (2013).

${ }^{5}$ M. Zwolak and M. D. Ventra, "Electronic signature of dna nucleotides via transverse transport," Nano Lett. 5, 421 (2005)

${ }^{6}$ M. Z. Bazant and T. M. Squires, "Induced-charge electrokinetic phenomena: Theory and microfluidic applications," Phys. Rev. Lett. 92, 066101 (2004).

${ }^{7}$ T. M. Squires and M. Z. Bazant, "Induced-charge electro-osmosis,” J. Fluid Mech. 509, 217 (2004)

${ }^{8}$ T. M. Squires, "Induced-charge electrokinetics: Fundamental challenges and opportunities," Lab Chip 9, 2477-2483 (2009).

${ }^{9}$ M. Z. Bazant, M. S. Kilic, B. D. Storey, and A. Ajdari, "Towards an understanding of induced-charge electrokinetics at large applied voltages in concentrated solutions," Adv. Colloid Interface Sci. 152, 48-88 (2009).

${ }^{10}$ T. M. Squires and M. Z. Bazant, "Breaking symmetries in induced-charge electro-osmosis and electrophoresis," J. Fluid Mech. 560, 65 (2006).

${ }^{11}$ H. Sugioka, "Microcolumn formation between electrodes in a narrow channel from metallic colloidal suspension through induced-charge electrophoresis," Phys. Rev. E 80, 016315 (2009).

${ }^{12}$ H. Sugioka, "High-speed rotary microvalves in water using hydrodynamic force due to induced-charge electrophoresis," Phys. Rev. E 81, 036301 (2010).

${ }^{13}$ A. Ramos, H. Morgan, N. G. Green, and A. Castellanos, "AC electric-field-induced fluid flow in microelectrodes," J. Colloid Interface Sci. 217, 420-422 (1999).

${ }^{14}$ A. Ramos, A. Gonzalez, A. Castellanos, N. G. Green, and H. Morgan, "Pumping of liquids with ac voltages applied to asymmetric pairs of microelectrodes," Phys. Rev. E 67, 056302 (2003).

${ }^{15} \mathrm{H}$. Sugioka, "Attitude and positioning control of elliptical particle using induced-charge electrophoresis for reading information," Colloids Surf., A 440, 27 (2014).

${ }^{16}$ H. Sugioka, "Basic analysis of induced-charge electrophoresis using the boundary element method," Colloids Surf., A 376, $102(2011)$.

${ }^{17}$ E. R. Weeks and H. L. Swinney, “Anomalous diffusion resulting from strongly asymmetric random walks," Phys. Rev. E 57, 4915 (1998).

${ }^{18}$ G. A. Schumacher and T. G. M. van de Ven, "Brownian motion of charged colloidal particles surrounded by electric double layers," Faraday Discuss. Chem. Soc. 83, 75-85 (1987).

${ }^{19} \mathrm{H}$. Zhao and H. Bau, "On the effect of induced electro-osmosis on a cylindrical particle next to a surface," Langmuir $\mathbf{2 3}$, 4053 (2007).

${ }^{20}$ M. Z. Bazant, K. Thornton, and A. Ajdari, “Diffuse-charge dynamics in electrochemical systems,” Phys. Rev. E 70, 021506 (2004).

${ }^{21}$ Y. Kalmykov, "Rotational Brownian motion in an external potential: The Langevin equation approach,” J. Mol. Liq. 69, $117-131$ (1996).

${ }^{22}$ T. M. Squires, "Effective pseudo-potentials of hydrodynamic origin,” J. Fluid Mech. 443, 403 (2001).

${ }^{23}$ K. Luo and R. Metzler, "Polymer translocation into a fluidic channel through a nanopore," Phys. Rev. E 82, 021922 (2010). 